\title{
紫茎泽兰种群年龄结构动态及对控制的指示意义
}

\author{
孙晓玉 ${ }^{1}$ 陆兆华 ${ }^{1}$ 于兴军 ${ }^{2}$ 桑卫国 ${ }^{2}$ \\ (1 中国矿业大学恢复生态学研究所, 北京 100083) \\ (2 中国科学院植物研究所植被数量生态学重点实验室, 北京 100093)
}

摘 要 外来入侵植物紫茎泽兰 (Eupatorium adenophorum) 给我国西南地区的经济、社会和环境造成了严重的危害。 为了阐明紫茎泽兰种群的年龄结构和未来发展趋势, 为今后的防治工作提供科学依据, 在攀枝花地区设置了 20 个 样地 $(10 \mathrm{~m} \times 10 \mathrm{~m})$, 对 4 种不同生境种群的年龄结构、静态生命表和时间序列预测进行了分析。这 4 个种群是该地 区有代表性类型中紫茎泽兰的种群: 路旁荒地、云南松 ( Pinus yunnanensis) 林、阔叶林和阔叶林的林缘。种群年龄结 构分析表明, 目前 (几个类型总和)个体数量比重分别为: 幼年期占 $92.32 \%$, 青年期占 $6.40 \%$, 成熟期占 $2.20 \%$, 衰 老期占 $0.18 \%$, 个体数量主要集中于幼年期。紫茎泽兰不同种群生命表和存活曲线的分析显示, 紫茎泽兰对 4 个 种群的入侵过程是路旁荒地 $\rightarrow$ 阔叶林林缘 $\rightarrow$ 阔叶林 $\rightarrow$ 云南松林, 尽管所处的生境差异较大, 但基本属于 Deevey III 型, 一年生幼苗死亡率高达 $97.30 \%$, 种群偏离典型曲线的程度与群落受干扰程度强弱有关, 一般由幼年期到青年 期和由成熟期到衰老期死亡率较高 (分别为 $93.07 \%$ 和 92.00\%)。时间序列分析表明, 在未来的 $3 \sim 5$ 年中, 紫茎泽 兰种群仍然是以青年期和成熟期个体占绝对优势。

关键词 紫茎泽兰 生命表 存活曲线 种群数量动态 时间序列

\section{AGE STRUCTURE DYNAMICS OF EUPATORIUM ADENOPHORUM POPULATIONS AND ITS IMPLICATIONS FOR CONTROL}

\author{
SUN Xiao-Yu ${ }^{1}$ LU Zhao-Hua ${ }^{1}$ YU Xing-Jun ${ }^{2}$ SANG Wei-Guo ${ }^{2 *}$ \\ (1 Institute of Restoration Ecology, China University of Mining and Technology, Beijing, 100083, China) \\ (2 Laboratory of Quantitative Vegetation Ecology, Institute of Botany, Chinese Academy of Sciences, Beijing 100093, China)
}

\begin{abstract}
The invasion of Eupatorium adenophorum has caused serious damage to natural ecosystems by suppressing native species populations in disturbed forests and pastures in southwest China. In this study, the age structure dynamics of $E$. adenophorum populations were examined in 20 plots $(10 \mathrm{~m} \times 10 \mathrm{~m})$ using the Greig-Smith grid method, in Panzhihua Prefecture, Sichuan Province of China. There is no standard method for identifying the age of an $E$. adenophorum plants, but through careful observations of the growth characteristics of E. adenophorum over several years in Panzhihua, we found a reliable method for aging individual plants. During the coldest season of every year, the apical half of branches grown in the current year wither and die, and a pair of opposite branches expand out from the lignified half that is below the wilted top. Although it can turn out clone genet alone once the branch touches the ground. At the same time sexual propagation and vegetative reproduction of radicicolous branches carry through from year to year. That is to say, the most number of branching ranks of the same branch is likely to indicate the real age of the plant. We dug out the entire plant in each grid and counted the grade rank of each branch to determine the age of each individual plant. The results showed that the age structure of the 4 populations were similar with most individuals in the infancy $(92.3 \%)$ and youth $(6.4 \%)$ periods. The analysis of life tables and survival curves showed that chronological sequence of an $E$. adenophorum invasion was as follows: first, invasion occurred along roadside fields, followed by invasion into the margins of broad-leaved forest, then deep into broad-leaved forest, and finally into Pinus yunnanensis forest. Even under different environmental conditions, survival curves of all $E$. adenophorum populations belonged to the Deevey type III pattern, and mortality of all populations showed a peak in 1-2 years old with mortality rates of $97.3 \%$. The degree to which a population deviated from a typical curve related to the intensity of human disturbance. In general, mortality during infancy period and mature period were high $(93.1 \%$ and $92.0 \%)$. The invasion time-sequence model predicts that young and mature individuals will dominate the populations at 3 and 5 years from the present. Therefore, in the Panzhihua Pre-
\end{abstract}


fecture, we predict that $E$. adenophorum will become a serious problem in 3 to 5 years. Compared with populations of other invasive plants, the population of $E$. adenophorum has a similar break out pattern among populations. Traits promoting weediness included the ability to reproduce sexually and asexually, rapid growth from seedling to sexual maturity, and, most importantly, adaptation to environmental stress (phenotypic plasticity) and high tolerance to environmental heterogeneity.

Key words Eupatorium adenophorum, Life table, Survival curve, Population dynamics, Time sequence

近些年来, 入侵种对本地种、群落及生态系统的 影响已得到广泛认知 (Elton, 1958; Lodge, 1993a, 1993b; Simberloff, 1996), 无数研究已经总结了入侵 种对本地种及群落结构的影响 (Williamson, 1996;

Wilcove et al., 1998; Parker et al., 1999; Sala et $a l$. 2000; Stein et al., 2000), 对入侵种在生态系统 水平上影响的研究也在进行中 (Vitousek \& Walker, 1989; Mooney \& Hobbs, 2000), 并被看作是全球变化 的主要内容之一(Vitousek et al., 1996)。

紫茎泽兰 (Eupatorium adenophorum) 原产中美洲, 现已在世界热带地区广泛分布。事实上, 这一物种对 世界许多地区构成严重危胁 (Patrabansh \& Madam, 1997; Oelrichs et al., 1998)。紫茎泽兰于 20 世纪 40 年代在我国云南南部被首次发现, 现广泛分布于云 南、广西、贵州、四川、西藏等省份。在其发生区常形 成单优群落, 排斥本地植物, 影响天然林的恢复, 侵入 经济林地和农田, 影响栽培植物生长, 堵塞水渠、阻碍 交通, 且植株有毒, 对畜牧业造成很大危害(李振宇和 谢炎,2002)。

为了更好地控制紫茎泽兰、减少其危害的程度 - 需要了解其种群的结构动态规律。种群的年龄结 构是指不同年龄组的个体在种群内的比例和配置情 况。研究种群的年龄结构对深入分析种群动态和进 行预测预报具有重要价值。生命表是反映种群全部
生活史的各年龄组或生活态级的出生率、死亡率等 综合信息表。它不仅能综合判断种群数量变化, 也 能反映出从出生到死亡的动态关系(李博等, 1999)。

\section{1 研究方法}

\section{1 样地调查}

本研究在四川省攀枝花市仁和区进行。在充分 踏查的基础上, 在路边荒地、阔叶林缘、云南松 ( Pinus yunnanensis) 林下、阔叶林下不同生境选取具有代 表性的地段作为调查样地, 分别设 $10 \mathrm{~m} \times 10 \mathrm{~m}$ 样地 20 块, 采用相邻格子法, 将每一块样地划分成 100 个 $1 \mathrm{~m} \times 1 \mathrm{~m}$ 的小样方。样地调查包括: 1)生境调 查: 包括地形地貌、海拔高度、坡位、坡向、土壤、及人 为干扰强度 (表 1);2) 群落学特征: 包括群落组成、 高度、盖度等; 3 ) 紫茎泽兰年龄的判定, 由于目前还 没有提出一个权威的标准, 我们根据紫茎泽兰的生 长特性(当年长出的枝干未木质化, 冬天会被冻坏, 来年在其两侧发出两个对生的新枝, 如此重复形成 庞大的株从), 结合当地人的判定方法, 采取用分枝 级数代表年龄的方法, 采取了将整棵大株挖出, 找出 其连根的主茎, 沿着主茎找出其上分枝级数最多的 一个侧枝, 从地上部分的梢部向地下部分根部数分 枝的级数, 所能查到的最大的分枝级数即为这株植 株的年龄。

表 1 不同紫茎泽兰种群的环境因子

Table 1 The environmental factors of different Eupatorium adenophorum populations

\begin{tabular}{|c|c|c|c|c|}
\hline $\begin{array}{c}\text { 环境因子 } \\
\text { Environmental factors }\end{array}$ & $\begin{array}{c}\text { 路旁荒地 } \\
\text { Roadside }\end{array}$ & $\begin{array}{c}\text { 阔叶林 } \\
\text { Broad-leaved forest }\end{array}$ & $\begin{array}{c}\text { 阔叶林林缘 } \\
\text { Margin of broad-leaved } \\
\text { forest } \\
\end{array}$ & $\begin{array}{c}\text { 云南松林 } \\
\text { Pinus yunnanensis forest }\end{array}$ \\
\hline 1 海拔 Altitude (m) & 1837 & 2175 & 2057 & 2208 \\
\hline 2 坡向 Slope aspect & 北 $\mathrm{N}$ & 西北 NW & 北 $\mathrm{N}$ & 西南 NS \\
\hline 3 坡度 Graduate $\left.{ }^{\circ}{ }^{\circ}\right)$ & 9 & 25 & 17 & 33 \\
\hline 4 坡位 Slope position & 下 Lower & 中 Middle & 中下 Mid-Lower & 上 Upper \\
\hline 5 乔木层盖度 Coverage of tree layer $\%$ ) & - & 70 & 60 & 50 \\
\hline 6 土壤厚度 Soil thickness $(\mathrm{cm})$ & 57 & 72 & 67 & 50 \\
\hline 7 土壤 $\mathrm{pH}$ 值 Soil $\mathrm{pH}$ & 6.0 & 6.1 & 6.0 & 5.8 \\
\hline 8 土壤水分含量 Soil moisture content $(\%)$ & 47 & 58 & 50 & 35 \\
\hline 9 种群平均密度 Mean density (individual $\bullet 10^{-2} \cdot \mathrm{m}^{-2}$ ) & 5133 & 863 & 2864 & 5108 \\
\hline 10 样地数量 No. of plots & 4 & 6 & 4 & 6 \\
\hline
\end{tabular}




\section{2 数据分析方法}

\subsection{1 年龄结构图绘制}

根据 1.1 中对紫茎泽兰年龄的判别方法, 统计 每一个样地内各年龄级紫茎泽兰的总株数, 以年龄 级为横坐标, 以同年龄株数 $\cdot 10^{-2} \cdot \mathrm{m}^{-2}$ 为纵坐标绘 制年龄结构图。刘伦辉等(1989)按照紫茎泽兰发育 阶段将其大致 4 个阶段: 幼年期 (1 2 年)、青年期 (3～6 年)、成熟期( $7 \sim 10$ 年)和衰老期( $12 \sim 15$ 年)。 据此, 本文将根据每个样地中紫茎泽兰种群划分阶 段。

\section{2 .2 静态生命表和存活曲线}

静态生命表是根据某一特定时间, 对种群作一 个年龄结构的调查, 并根据其结果编制而成。各年 龄段的 $n_{x}$ 为 $x$ 期开始时的存活数, 由原测定数据给 出。

$x$ 到 $x+1$ 的死亡数

$$
d_{x}=n_{x}-n_{x+1}
$$

$x$ 到 $x+1$ 的死亡率

$q_{x}=d_{x} / n_{x}$

$x$ 到 $x+1$ 期的平均存活数 $L_{x}=\left(n_{x}+n_{x+1}\right) / 2$

进入 $x$ 龄期的全部个体在进入 $x$ 期以后的存活 个体总年数, 即 $T_{x}=\sum L_{x}$

如 $T_{0}=L_{0}+L_{1}+L_{2}+\cdots ; T_{1}=L_{1}+L_{2}+L_{3}+$

\section{生命期望 $\quad e_{x}=T_{x} / n_{x}$}

具体方法见文献(李博等, 1999)。

以平均寿命的百分比表示年龄 $x$, 作横坐标, 存 活 $L_{x}$ 的对数作纵坐标, 画成存活曲线图。再根据曲 线比较属于那种类型。

1.2.3 种群数量动态的时间序列预测模型

利用生命表进行时间序列预测的计算公式为:

$m_{n}=\left(n_{1}+n_{2}+\cdots+n_{n}\right) / n$

式中: $m_{n}$ 为所预测的 $n$ 年后的植株数; $n_{x}$ 为 $x$ 期开始时的存活数(张文辉, 1998)。本文对未来 3
年和 5 年的种群发展趋势进行预测。

\section{2 结果与分析}

\section{1 不同紫茎泽兰种群的年龄结构}

图 1a 是 4 个种群紫茎泽兰 1 年生幼苗的个体 数。在 4 种类型的样地中, 1 年生幼苗的平均个体 数分别是: 1) 阔叶林 616 个;2) 阔叶林林缘 2764 个; 3)云南松林 4796 个; 4) 路旁荒地 4355 个。图 1b $\mathrm{e}$ 分别是阔叶林、阔叶林林缘、云南松林、路边荒地 各种群紫茎泽兰年龄结构图。图 $1 \mathrm{~b}$ 为阔叶林中紫 茎泽兰种群年龄结构。该阔叶林为常绿次生阔叶 林, 主要优势种是樟科石楠属 (Photinia)、壳斗科石 栎属( Lithocarpus)、楉属 (Castanopsis) 植物。图 1c 是 阔叶林林缘紫茎泽兰种群年龄结构。图 1d 为纯云 南松林中紫茎泽兰种群年龄结构。图 1e 是路边荒 地紫茎泽兰种群年龄结构。图 1f 为将 4 个类型的 紫茎泽兰种群汇总以后的年龄结构图 (总和)。从结 构图中可以看出, 攀枝花紫茎泽兰种群幼年期 (1 2 年) 个体数量比重占 $92.32 \%$; 青年期(3６年)个体 数量比重占 $6.40 \%$; 成熟期 (7～11 年) 个体数量比 重占 $2.20 \%$; 衰老期 (12 15 年) 个体数量比重占 $0.18 \%$, 目前种群仍处于增长阶段。

\section{2 紫茎泽兰种群生命表分析}

阔叶林、阔叶林林缘、云南松林及路旁荒地 4 种 生境紫茎泽兰种群的静态生命表分别见表 2、表 3、 表 4 和表 5。从表中可以看出, 4 种生境的紫茎泽兰 最大年龄是不同的,阔叶林中紫茎泽兰的最大年龄 是 8 年, 阔叶林林缘为 9 年, 云南松林中为 7 年, 而 路旁荒地则为 15 年。说明该 4 种生境紫茎泽兰入 侵的先后顺序是: 路旁荒地 (15 年) $\rightarrow$ 阔叶林林缘 (9 年) $\rightarrow$ 阔叶林 ( 8 年) $\rightarrow$ 云南松林 $(7$ 年)。紫茎泽兰在 年龄为 1 时有较高的死亡率, 说明其发育初期, 个体

表 2 阔叶林中紫茎泽兰种群生命表

Table 2 The life table of Eupatorium adenophorum population in broad-leaved forest

\begin{tabular}{|c|c|c|c|c|c|c|}
\hline $\begin{array}{c}\text { 年龄 }(X) \\
\text { Age }\end{array}$ & $\begin{array}{c}\text { 存活数 }\left(N_{X}\right) \\
\text { Number of survival }\end{array}$ & $\begin{array}{c}\text { 存活数标准化 }\left(L_{X}\right) \\
\text { Standard survival }\end{array}$ & $\operatorname{LOG} L_{X}$ & $\begin{array}{c}\text { 死亡数 }\left(D_{X}\right) \\
\text { Standard death at interval }\end{array}$ & $\begin{array}{l}\text { 死亡率 }\left(Q_{X}\right) \\
\text { Mortality rate }\end{array}$ & $\begin{array}{c}\text { 生命期望 }\left(E_{X}\right) \\
\text { Expecting life }\end{array}$ \\
\hline 1 & 616 & 1000 & 3.000 & 513 & 0.833 & 0.901 \\
\hline 2 & 103 & 167.210 & 2.223 & 34 & 0.330 & 1.898 \\
\hline 3 & 69 & 112.010 & 2.049 & 21 & 0.304 & 1.587 \\
\hline 4 & 48 & 77.920 & 1.892 & 31 & 0.646 & 1.063 \\
\hline 5 & 17 & 27.590 & 1.441 & 14 & 0.824 & 1.088 \\
\hline 6 & 3 & 4.870 & 0.688 & - & - & 2.833 \\
\hline 7 & 4 & 6.490 & 0.812 & 1 & 0.250 & 1.250 \\
\hline 8 & 3 & 4.870 & 0.688 & 3 & 1.000 & 0.500 \\
\hline
\end{tabular}




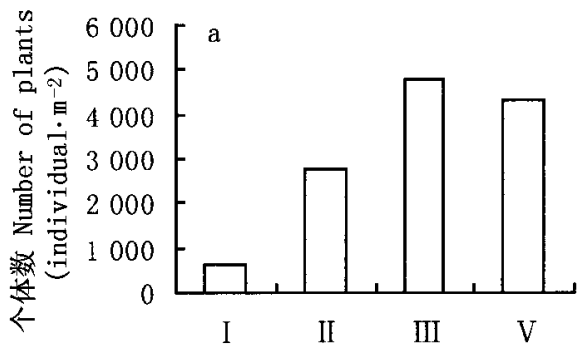

各样地类型 Plot type
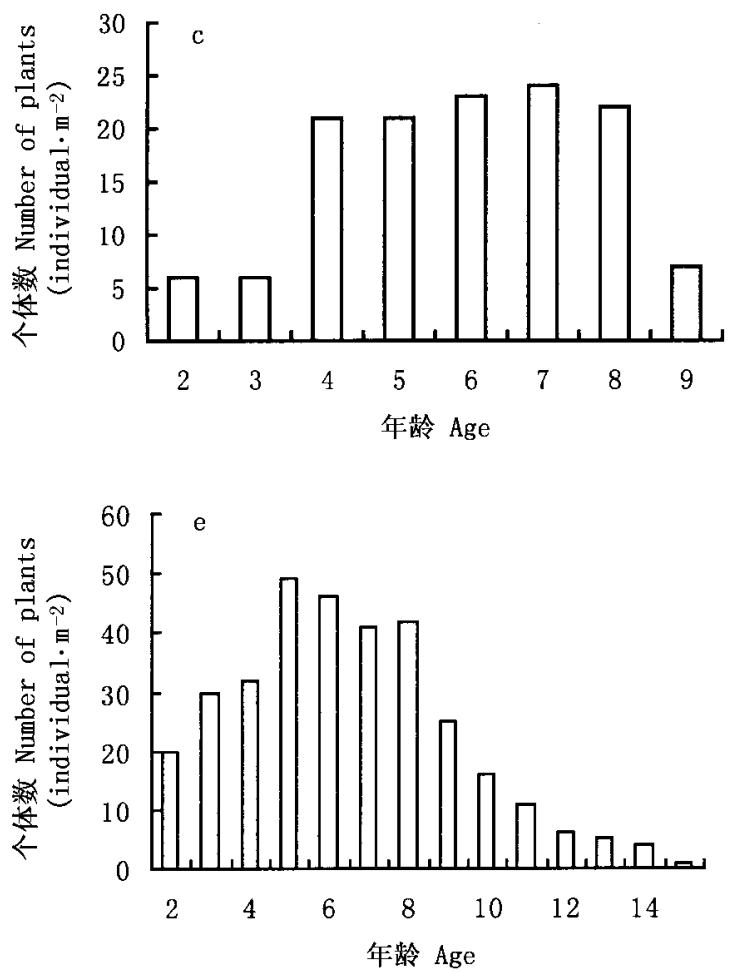
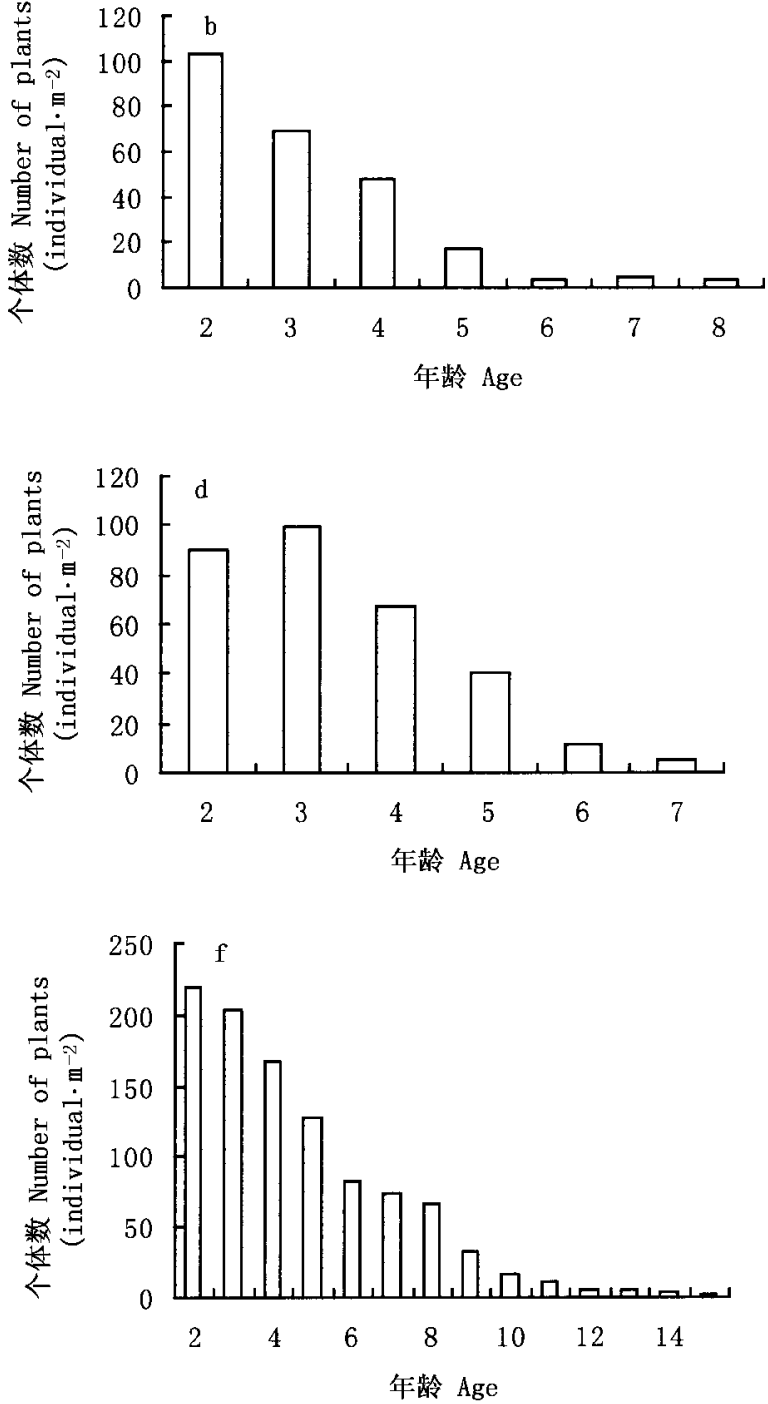

图 1 各种群年龄结构

Fig.1 The age structure of different Eupatorium adenophorum populations

a. 各样地中一年生紫茎泽兰植株 b. 阔叶林紫茎泽兰种群 c. 阔叶林林缘紫茎泽兰种群 d. 云南松林紫茎泽兰种群 e. 路旁荒地紫 茎泽兰种群 f. 各种群(总和) I. 阔叶林一年生紫茎泽兰苗个体总数 II. 阔叶林林缘一年生紫茎泽兰个体总数 III. 云南松林一年生紫 茎泽兰个体总数 IV. 路旁荒地一年生紫茎泽兰个体总数 a. One-year-old Eupatorium adenophorum plants of different populations b. The populations of $E$. adenophorum in broad-leaved forest c. The population of $E$. adenophorum at the margin of broad-leaved forest d. The population of $E$. adenophorum in Pinus yunnanensis forest e. The population of $E$. adenophorum in roadside field $\mathrm{f}$. The total populations I . The total individuals of oneyear-old E. adenophorum in broad-leaved forest II . The total individuals of one-year-old E. adenophorum at the margin of broad-leaved forest III . The total individuals of one-year-old E. adenophorum in Pinus yunnanensis forest IV . The total individuals of one-year-old E. adenophorum in roadside field

数目极其丰富, 如在云南松林紫茎泽兰幼苗为 4796 棵,而 2 年生紫茎泽兰个体数达到 90 , 死亡率为 $98.12 \%$ 。可见由于种内、种间竞争和群落所在环境 条件的作用, 从年龄 1 到 2 阶段, 个体数目急剧减 小。紫茎泽兰是一种竞争型杂草, 在生长初期大量繁 殖, 以在新生境中为自己存活创造机会。这样与其它 物种同时迁移到新生境中时,利用自己的高生产力, 占据受干扰的场所。虽然它的幼苗的存活率不大, 但
只要成为 2 年以上植株, 就会稳定下来, 继续大量繁 殖新植株, 泛滥成灾。所以, 它在初期是 $r$-对策选择 者, 青年期后就是 $\mathrm{K}$-对策选择为主, $r$-对策选择为辅。

\section{3 各种群存活曲线}

以 4 种生境中紫茎泽兰种群生命表中的年龄为 横坐标, 以 $\mathrm{LOG} L_{X}$ 为纵坐标绘制了 4 个种群的存活 曲线(图 2)。紫茎泽兰种群的存活曲线基本属于 Deevey III 型。图2直观地反映了不同生境中紫茎泽 
表 3 阔叶林林缘紫茎泽兰种群生命表

Table 3 The life table of Eupatorium adenophorum population in the margin of broad-leaved forest

\begin{tabular}{|c|c|c|c|c|c|c|}
\hline $\begin{array}{c}\text { 年龄 }(X) \\
\text { Age }\end{array}$ & $\begin{array}{c}\text { 存活数 }\left(N_{X}\right) \\
\text { Number of survival }\end{array}$ & $\begin{array}{c}\text { 存活数标准化 }\left(L_{X}\right) \\
\text { Standard survival }\end{array}$ & $\operatorname{LOG} L_{X}$ & $\begin{array}{c}\text { 死亡数 }\left(D_{X}\right) \\
\text { Standard death at interval }\end{array}$ & $\begin{array}{l}\text { 死亡率 }\left(Q_{X}\right) \\
\text { Mortality rate }\end{array}$ & $\begin{array}{c}\text { 生命期望 }\left(E_{X}\right) \\
\text { Expecting life }\end{array}$ \\
\hline 1 & 2764 & 1000.000 & 3.000 & 2758 & 0.998 & 0.547 \\
\hline 2 & 6 & 2.171 & 0.337 & 0 & 0 & 21.170 \\
\hline 3 & 6 & 2.171 & 0.337 & - & - & 20.170 \\
\hline 4 & 21 & 7.598 & 0.881 & 0 & 0 & 5.119 \\
\hline 5 & 21 & 7.598 & 0.881 & - & - & 4.119 \\
\hline 6 & 23 & 8.321 & 0.92 & - & - & 2.804 \\
\hline 7 & 24 & 8.683 & 0.939 & 2 & 0.083 & 1.708 \\
\hline 8 & 22 & 7.960 & 0.901 & 15 & 0.682 & 0.818 \\
\hline 9 & 7 & 2.533 & 0.404 & 7 & 1.000 & 0.500 \\
\hline
\end{tabular}

表 4 云南松林紫茎泽兰种群生命表

Table 4 The life table of Eupatorium adenophorum population in Pinus yunnanensis forest

\begin{tabular}{ccccccc}
\hline $\begin{array}{c}\text { 年龄 }(X) \\
\text { Age }\end{array}$ & $\begin{array}{c}\text { 存活数 }\left(N_{X}\right) \\
\text { Number of survival }\end{array}$ & $\begin{array}{c}\text { 存活数标准化 }\left(L_{X}\right) \\
\text { Standard survival }\end{array}$ & LOG $L_{X}$ & $\begin{array}{c}\text { 死亡数 }\left(D_{X}\right) \\
\text { Standard death at interval }\end{array}$ & $\begin{array}{c}\text { 死亡率 }\left(Q_{X}\right) \\
\text { Mortality rate }\end{array}$ & $\begin{array}{c}\text { 生命期望 }\left(E_{X}\right) \\
\text { Expecting life }\end{array}$ \\
\hline 1 & 4796 & 1000.000 & 3.000 & 4706 & 0.981 & - \\
2 & 90 & 18.766 & 1.273 & - & 0.323 & 2.967 \\
3 & 99 & 20.642 & 1.315 & 32 & 0.403 & 1.742 \\
4 & 67 & 13.970 & 1.145 & 27 & 0.725 & 0.546 \\
5 & 40 & 8.340 & 0.921 & 6 & 0.900 \\
6 & 11 & 2.294 & 0.361 & 5 & 1.000 & 0.955 \\
7 & 5 & 1.043 & 0.018 & 538.000 & 000 \\
\hline
\end{tabular}

表 5 路旁荒地紫茎泽兰种群生命表

Table 5 The life table of Eupatorium adenophorum population in roadside field

\begin{tabular}{|c|c|c|c|c|c|c|}
\hline $\begin{array}{c}\text { 年龄 }(X) \\
\text { Age }\end{array}$ & $\begin{array}{c}\text { 存活数 }\left(N_{X}\right) \\
\text { Number of survival }\end{array}$ & $\begin{array}{c}\text { 存活数标准化 }\left(L_{X}\right) \\
\text { Standard survival }\end{array}$ & $\operatorname{LOG} L_{X}$ & $\begin{array}{c}\text { 死亡数 }\left(D_{X}\right) \\
\text { Standard death at interval }\end{array}$ & $\begin{array}{l}\text { 死亡率 }\left(Q_{X}\right) \\
\text { Mortality rate }\end{array}$ & $\begin{array}{c}\text { 生命期望 }\left(E_{X}\right) \\
\text { Expecting life }\end{array}$ \\
\hline 1 & 4355 & 1000.000 & 3.000 & 4335 & 0.995 & 0.575 \\
\hline 2 & 20 & 4.592 & 0.662 & - & - & 15.900 \\
\hline 3 & 30 & 6.889 & 0.838 & - & - & 9.767 \\
\hline 4 & 32 & 7.348 & 0.866 & - & - & 8.188 \\
\hline 5 & 49 & 11.251 & 1.051 & 3 & 0.061 & 4.520 \\
\hline 6 & 46 & 10.563 & 1.024 & 5 & 0.109 & 3.783 \\
\hline 7 & 41 & 9.415 & 0.974 & - & - & 3.183 \\
\hline 8 & 42 & 9.644 & 0.984 & 17 & 0.405 & 2.119 \\
\hline 9 & 25 & 5.741 & 0.759 & 9 & 0.360 & 2.220 \\
\hline 10 & 16 & 3.674 & 0.565 & 5 & 0.313 & 2.188 \\
\hline 11 & 11 & 2.526 & 0.402 & 5 & 0.455 & 1.955 \\
\hline 12 & 6 & 1.378 & 0.139 & 1 & 0.167 & 2.167 \\
\hline 13 & 5 & 1.148 & 0.060 & 1 & 0.200 & 1.500 \\
\hline 14 & 4 & 0.919 & -0.040 & 3 & 0.750 & 0.750 \\
\hline 15 & 1 & 0.230 & -0.640 & 1 & 1.000 & 0.500 \\
\hline
\end{tabular}

兰种群数量动态特征: 1) 在各个种群中, 幼年期个体 数量庞大, 老年期数量少, 阔叶林、阔叶林林缘及路 旁荒地 3 个种群还正值种群增长的旺盛时期。2) 4 个种群虽然存在环境条件上的差异, 但种群死亡率 高峰都出现在 1 年向 2 年过渡期间, 2 年以后各年 龄级间的死亡率基本比较稳定。3）云南松林下灌 木和草本植物的物种数量较阔叶林下稀少, 厚厚的 松针层可以为紫茎泽兰种子的萌发以及幼苗早期的 喜荫性提供了条件, 因此云南松林中紫茎泽兰种群 的密度最大; 阔叶林林缘中紫茎泽兰种群由于乔、灌
木层荫蔽度较高, 物种组成较丰富, 天保工程实施后 受人为干扰强度相对较小, 因此种群密度小, 由林缘 向阔叶林中渐次发展密度逐渐变小, 而路旁荒地受 紫茎泽兰入侵的时间最长, 经历了激烈的种内、种间 竞争, 种群发展趋于平缓, 种群密度较之初期有所降 低。

2.44 种生境类型紫茎泽兰种群数量动态的时间 序列预测分析

以生命表的内容为原始数据, 按照一次平均推 移法计算出攀枝花市仁和区 4 种生境类型种群数量 


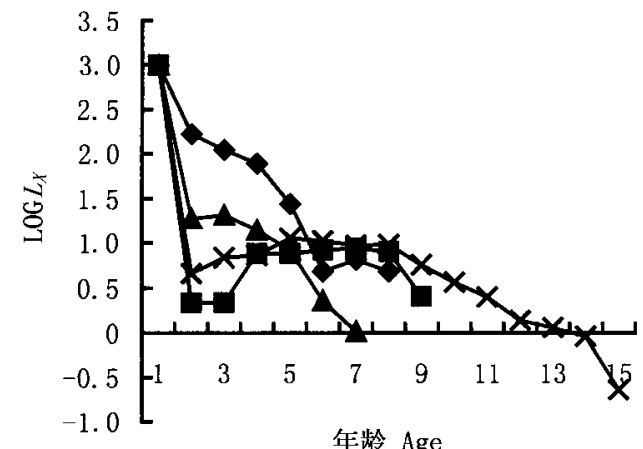

年龄 Age

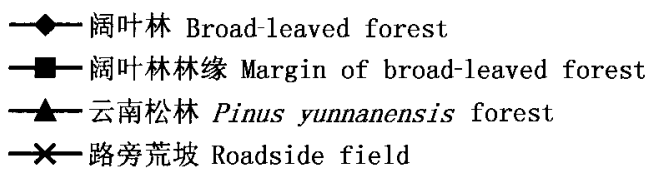

图 24 种生境下紫茎泽兰存活曲线图

Fig. 2 Survival curves of different Eupatorium adenophorum populations

动态在未来 3 年、 5 年的株数, 结果见表 $6 \sim 9$ 。从表 6 9 中可以看出, 紫茎泽兰各种群的各年龄株数峰 值在预测序列中依次向后推移。依据现有种群中紫 茎泽兰个体的数量, 在 3 年以后, 在阔叶林紫茎泽兰 种群中, 青年期比例占 $97.27 \%$, 成熟期比例占 $2.81 \%$; 在阔叶林林缘紫茎泽兰种群中, 青年期比例 占 $93.80 \%$, 成熟期比例占 $6.11 \%$; 在云南松林紫茎 泽兰种群中, 青年期比例占 $99.00 \%$, 成熟期比例占 $1.00 \%$; 在路旁荒地紫茎泽兰种群中, 青年期比例占 $88.93 \%$, 成熟期比例占 $9.56 \%$, 衰老期比例占 $1.51 \%$ 。而在未来 5 年以后, 在阔叶林紫茎泽兰种群 中, 青年期比例占 $80.43 \%$, 成熟期比例占 $19.57 \%$ ； 在阔叶林林缘紫茎泽兰种群中, 青年期比例占 $90.53 \%$, 成熟期比例占 $9.47 \%$; 在云南松林紫茎泽 兰种群中, 青年期比例占 $96.05 \%$, 成熟期比例占 $3.95 \%$; 在路旁荒地紫茎泽兰种群中, 青年期比例占

表 6 阔叶林紫茎泽兰生命预测表

Table 6 Time sequence prediction of quantitative dynamics of Eupatorium adenophorum in broad-leaved forest

\begin{tabular}{cccc}
\hline$X$ & $N_{X}$ & $\begin{array}{c}\text { 未来 } 3 \text { 年的株数(M3) } \\
\text { No. of plants after } \\
\text { three years }\end{array}$ & $\begin{array}{c}\text { 未来 } 5 \text { 年的株数 (M5) } \\
\text { No. of plants after } \\
\text { five years }\end{array}$ \\
\hline 1 & 616 & & \\
2 & 103 & & \\
3 & 69 & 262.67 & \\
4 & 48 & 73.33 & 170.60 \\
5 & 17 & 44.67 & 48.00 \\
6 & 3 & 22.67 & 28.20 \\
7 & 4 & 8.00 & 25.00 \\
8 & 3 & 3.33 & \\
\hline
\end{tabular}

表 7 阔叶林林缘紫茎泽兰生命预测表

Table 7 Time sequence prediction of quantitative dynamics of Eupatorium adenophorum in the margin of broad-leaved forest

\begin{tabular}{cccc}
\hline$X$ & $N_{X}$ & $\begin{array}{c}\text { 未来 3 年的株数(M3) } \\
\text { No. of plants after } \\
\text { three years }\end{array}$ & $\begin{array}{c}\text { 未来 } 5 \text { 年的株数 (M5) } \\
\text { No. of plants after } \\
\text { five years }\end{array}$ \\
\hline 1 & 2764 & & \\
2 & 6 & & \\
3 & 6 & 925.33 & \\
4 & 21 & 11.00 & 563.60 \\
5 & 21 & 16.00 & 15.40 \\
6 & 23 & 21.67 & 19.00 \\
7 & 24 & 22.67 & 22.20 \\
8 & 22 & 23.00 & 19.40 \\
9 & 7 & 17.67 & \\
\hline
\end{tabular}

$X 、 N_{X}$ : 同表 2 See Table 2

表 8 云南松林紫茎泽兰生命预测表

Table 8 Time sequence prediction of quantitative dynamics of Eupatorium adenophorum in Pinus yunnanensis forest

\begin{tabular}{cccc}
\hline$X$ & $N_{X}$ & $\begin{array}{c}\text { 末来 } 3 \text { 年的株数 (M3) } \\
\text { No. of plants after } \\
\text { three years }\end{array}$ & $\begin{array}{c}\text { 未来 } 5 \text { 年的株数 (M5) } \\
\text { No. of plants after } \\
\text { five years }\end{array}$ \\
\hline 1 & 4796 & & \\
2 & 90 & 1661.67 & \\
3 & 99 & 85.33 & 1018.40 \\
4 & 67 & 68.67 & 61.40 \\
5 & 40 & 39.33 & 44.40 \\
6 & 11 & 18.67 & \\
7 & 5 & & \\
\hline
\end{tabular}

$X 、 N_{X}$ : 同表 2 See Table 2

表 9 路旁荒地紫茎泽兰生命预测表

Table 9 Time sequence prediction of quantitative dynamics of Eupatorium adenophorum in roadside field

\begin{tabular}{cccc}
\hline$X$ & $N_{X}$ & $\begin{array}{c}\text { 未来 } 3 \text { 年的株数 (M3) } \\
\text { No. of plants after } \\
\text { three years }\end{array}$ & $\begin{array}{c}\text { 未来 } 5 \text { 年的株数 (M5) } \\
\text { No. of plants after } \\
\text { five years }\end{array}$ \\
\hline 1 & 4355 & & \\
2 & 20 & 1468.33 & \\
3 & 30 & 27.33 & \\
4 & 32 & 37.00 & 897.20 \\
5 & 49 & 42.33 & 35.40 \\
6 & 46 & 45.33 & 39.60 \\
7 & 41 & 43.00 & 42.00 \\
8 & 42 & 36.00 & 40.60 \\
9 & 25 & 27.67 & 34.00 \\
10 & 16 & 17.33 & 27.00 \\
11 & 11 & 11.00 & 20.00 \\
12 & 6 & 7.33 & 12.60 \\
13 & 5 & 5.00 & 8.40 \\
14 & 4 & 3.33 & 5.40 \\
15 & 1 & &
\end{tabular}

$X 、 N_{X}$ : 同表 2 See Table 2

$80.24 \%$, 成熟期比例占 $15.76 \%$, 衰老期比例占 $3.99 \%$ 。总体上来说, 未来 $3 \sim 5$ 年后, 种群仍然是 以青年期个体占绝对优势, 因而种群的危害将仍然 十分严重。 


\section{3 讨 论}

\section{1 紫茎泽兰种群基本特征}

紫茎泽兰种群结构特征的幼年阶段个体较丰 富, 成年阶段数量基本稳定, 表明其幼年阶段的个体 向成年阶段的发育是不连续的。幼株生长过程中, 云南松林中的紫茎泽兰在 2 年龄段,阔叶林林缘的 在 $3 、 5$ 和 6 年龄段, 阔叶林下的在 6 年龄段, 荒坡 $2 、$ 4 和 7 这几个年龄段, 可能经过某种自然灾害或人 为等环境篎选, 导致这些阶段数量较少或缺失, 使计 算死亡率和存活率时出现间隔, 少量个体承受住了 环境的笁选,完成了整个种群生活史。种群生态对 策从倾向于 $r$-对策转变为倾向于 $\mathrm{K}$-对策, 说明入侵 生物在对不同的新生境入侵的过程中, 并不单纯依 赖单一的生态对策, 而是可以通过多种生态对策来 实现。

几种生境下的紫茎泽兰存活曲线均为凹线型, 也即 Deevey 划分的存活曲线类型中的 III 型。存活 曲线直观地表达了攀枝花 4 种生境下的紫茎泽兰种 群的存活过程, 紫茎泽兰幼年期死亡率很大, 第二年 后每年的死亡率基本比较恒定。

3.2 紫茎泽兰种群年龄结构及动态的时间序列预 测分析

根据紫茎泽兰种群 (总和) 年龄结构, 种群 (总 和) 个体数量比重分别为: 幼年期占个体总数的 $92.32 \%$, 青年期占个体总数的 $6.40 \%$, 成熟期占个 体总数的 $2.20 \%$, 衰老期占个体总数的 $0.18 \%$, 目 前种群数量主要集中于幼年期。

从 4 种生境类型的紫茎泽兰年龄结构时间序列 预测表中可以看出, 紫茎泽兰种群各年龄除个别年 龄外, 未来 3 年和 5 年均呈增加势态, 随年龄增加各 年龄级株数增幅差异较大, 以云南松林为例, 3 年后 青年期个体占种群总数的 $98.98 \%$ ，成熟期个体占 $1.02 \% ; 5$ 年后青年期个体占 $96.05 \%$, 成熟期个体 占 $3.95 \%$ 。此前, 有人提出紫茎泽兰是一种过渡性 的先锋群落, 任其自然发展也必将被淘汰(刘伦辉 等, 1989)。根据本研究结果分析, 至少未来 5 年 内,现在的 4 种生境类型依然是被旺盛生长的青年 期群落所占据,因此会形成极大的危害。

$$
\text { 参 考 文 献 }
$$

Elton CS (1958). The Ecology of Invasions by Animals and Plants.
Methuen, London, 181.

Li B (李博), Yang C (杨持), Lin P (林鹏) (1999). Ecology (生态学). Higher Education Press, Beijing, 43. (in Chinese) Li ZY (李振宇), Xie Y (解炎) (2002). Invasive Alien Species in China (中国外来入侵种). China Forestry Publishing House, Beijing, 163. (in Chinese)

Liu LH (刘伦辉)，Liu WY (刘文耀)，Zheng Z (郑征)，Jing GF (荆桂芬) (1989). The characteristic research of autecology ecology of Pamakani (Eupatorium adenophorum). Acta Ecological Sinica (生态学报), 5, 1-6. (in Chinese with English abstract)

Lodge DM (1993a). Biological invasions: lessons for ecology. Trends in Ecology \& Evolution, 8, 133 - 137.

Lodge DM (1993b). Species invasions and deletions: community effects and responses to climate and habitat change. In: Karieva PM, Kingsolver JG, Huey RB eds. Biotic Interactions and Global Change. Sinauer Associates, Sumderland, MA, 367 - 387.

Mooney HA, Hobbs RJ (2000). Invasive Species in a Changing World. Island Publishing House, Washington, DC, 457.

Oelrichs PB, Seawright AA, MacLeodJ JK, Ng JC (1998). Toxic Plants and Other Natural Toxicants. CAB International, Wallingford, UK, 271.

Parker IM, Simberloff D, Lonsdale WM, Goodell K, Wonham M (1999). Impact: toward a framework for understanding the ecological effects of invaders. Biological Invasions, 1, 3-19.

Patrabansh S, Madan M (1997). Studies on cultivation, biological efficiency and chemical analysis of Pleurotus sajor-caju (FR.) SINGER on different bio-wastes. Acta Biotechnologica, 17, 107 -122 .

Sala OE, Chapin FS, Armesto JJ, Berlow E, Bloomfield J (2000) . Global spread of microorganisms by ships. Nature, 408, 49 50 .

Simberloff D (1996). Impacts of introduced species in the United States. Consequences, 2, $13-222$.

Stein B, Kutner LS, Adams JS (2000). Precious Heritage: the Status of Biodiversity in the United States. Oxford University Press, London, 300.

Vitousek PM, Antonio CMD, Loope LL, Westbrooks R (1996). Biological invasions as global environmental change. American Scientist, 84, $468-478$.

Vitousek PM, Walker LR (1989). Biological invasion by Myrica faya in Hawaii: plant demography, nitrogen fixation, ecosystem effects. Ecological Monographs, 59, 247 - 265.

Williamson M (1996). Biological Invasions. Chapman \& Hall, New York, 244.

Wilcove DS, Rothstein D, Dubow J, Phillips A, Losos E (1998). Quantifying threats to imperiled species in the United States. BioScience, 48, 607-615.

Zhang WH (张文辉) (1998). Study on the Population Ecology of Adenophora potanini (裂叶沙参种群生态学研究). The Publishing House of Northeast Forestry University, Harbin. (in Chinese) 